\title{
GEINTEC
}

\section{TERPENOS COM APLICAÇÃO CARDIOVASCULAR}

\section{TERPENE WITH CARDIOVASCULAR APLICATION}

Américo Azevedo de Souza ${ }^{1}$; Sheyla Alves Rodrigues ${ }^{2}$; José Evaldo Rodrigues de Menezes Filho ${ }^{3}$; Carla Maria Lins de Vasconcelos ${ }^{4}$; Mairim Russo Serafini ${ }^{5}$; Lucindo José Quintans Júrnior ${ }^{6}$;

Charles dos Santos Estevam ${ }^{7}$

${ }^{1}$ Universidade Federal de Sergipe - UFS - São Cristóvão/SE - Brasil americosouza_bio@yahoo.com.br

${ }^{2}$ Instituto Federal de Sergipe - IFS - Itabaiana/SE - Brasil

shelrodrigues@hotmail.com

${ }^{3}$ Universidade Federal de Sergipe - UFS - São Cristóvão/SE - Brasil menezesfilho10@yahoo.com.br

${ }^{4}$ Universidade Federal de Sergipe - UFS - São Cristóvão/SE - Brasil carlamlv@hotmail.com

${ }^{5}$ Universidade Federal de Sergipe - UFS - São Cristóvão/SE - Brasil mairserafini@hotmail.com

${ }^{6}$ Universidade Federal de Sergipe - UFS - São Cristóvão/SE - Brasil lucindo_jr@yahoo.com.br

${ }^{7}$ Universidade Federal de Sergipe - UFS - São Cristóvão/SE - Brasil cse.ufs@gmail.com

\section{Resumo}

O uso de plantas medicinais como alternativa terapêutica é tão antigo quanto a própria existência humana. Desta maneira, a procura por moléculas com efetividade para aplicações cardiovasculares é uma alternativa para as pesquisas farmacológicas. Nesta gama de moléculas estão inseridos os terpenos, constituintes de óleos essenciais que estão contidos em todos os órgãos das plantas e que apresentam diversas aplicações farmacológicas, inclusive propriedades cardiovasculares. Assim, o objetivo desse trabalho foi realizar uma triagem nos depósitos de patentes sobre a aplicação cardiovascular de terpenos até o momento. Para isso, a prospecção foi realizada no Escritório Europeu de Patentes, na Organização Mundial de Propriedade Intelectual, no Escritório Americano de Marcas e Patentes e no Instituto Nacional de Propriedade Industrial do Brasil. O maior número de pedidos de patentes encontrados foi no Escritório Americano de Marcas e Patentes. Os maiores depositantes foram Estados Unidos e China. Os maiores requerentes de propriedade intelectual foram empresas e pessoas físicas. $O$ Brasil não apresentou pedido de registro de patente. A classificação internacional que mais ocorreu nessa prospecção foi a A61K.

Palavras-chave: terpenos; cardiovascular; prospecção tecnológica; patentes. 


\begin{abstract}
The use of medicinal plants as alternative therapy is as old as human existence itself. Thus, the search for molecules with effectiveness for cardiovascular applications is an alternative to pharmacological research. In this range of molecules are inserted terpenes, constituents of essential oils which are contained in all plant organs and that exhibit various pharmacological applications including cardiovascular properties. Thus, the aim of this study was to perform a screening in patent applications on cardiovascular application of terpenes yet. For this, the prospect was held at the European Patent Office, the World Intellectual Property Organization, the U.S. Patent and Trademark Office and the National Institute of Industrial Property of Brazil. The largest number of patent applications has been found in U.S. Patent and Trademark Office. The largest depositors were the United States and China. The largest intellectual property applicants were companies and individuals. Brazil has not filed a patent registration. The international classification that occurred over this prospect was A61K.
\end{abstract}

Key-words: terpenes, cardiovascular, technological forecasting; patents.

\title{
1. Introdução.
}

Doenças cardiovasculares são responsáveis por acometer uma grande quantidade de pessoas mundialmente (GIELEN et al., 2014, p.307). Em 2010, um estudo relatado pela Organização Mundial de Saúde (OMS) demonstrou que a quantidade de casos de patologias cerebrovasculares e isquemias cardíacas tem aumentado significativamente desde um último estudo bastante similar realizado em 1990, onde estas patologias apresentam-se atualmente no topo das principais causas de mortalidade no mundo (LOZANO et al., 2012, p. 2097). Por este motivo existe uma busca efetiva de novos fármacos para o tratamento de doenças cardiovasculares.

Ao longo da evolução científica, alternativas terapêuticas bastante eficazes tem sido descobertas, tais como a digoxina encontrada nas raízes de Digitalis spp, demonstrado assim a contribuição efetiva no interesse em explorar plantas medicinais com propriedades cardioativas, especialmente para tratar doenças cardíacas atuais (AKINMOLADUN., et al, 2014., p. 396).

Sabe-se que a utilização de plantas medicinais tem aumentado progressivamente em todo o mundo devido a fatores como facilidade de utilização e aquisição (EDDOUKS et al., 2012, p.2). Um dos fatores atribuídos ao efeito terapêutico desempenhado pelas plantas medicinais é relacionado à presença de compostos conhecidos quimicamente como terpenos que são encontrados em cerca de $90 \%$ dos óleos essenciais de diversos órgãos das plantas tais como raízes, caules, folhas flores e sementes, bem como na casa e entrecasca de caules (ALMEIDA et al., 2011, p. 2727).

Os terpenos são moléculas de tamanho relativamente pequeno, os quais apresentam em sua constituição seguimentos de unidades isoprênicas com átomos de carbono geralmente múltiplos de cinco ligados entre si (MATICH et al., 2007, p. 1748). Apesar de serem moléculas estruturalmente 
simples, são atribuídas várias atividades farmacológicas aos terpenos (CERQUEIRA et al., 2011, p. 138; SANTOS et al., 2011, p. 764; SIQUEIRA et al., 2006,. p. 2365).

Acerca dos efeitos farmacológicos apresentados pelos terpenos destacam-se as atividades no sistema cardiovascular. Diversos autores relatam o potencial de terpenos em ensaios farmacológicos onde estes desempenham atividades vasorelaxante, hipotensiva e na diminuição da frequência cardíaca (PEIXOTO-NEVES et al., 2010, p. 342; BASTOS et al., , 2010, p. 331; AYDINET al., 2007, p. 1368; MAGALHÃES et al., 2008, p. 174). Desta forma justifica-se a utilização de terpenos para a prevenção ou tratamento de patologias cardiovasculares.

Assim o objetivo do presente trabalho foi investigar os registros de patentes referentes à utilização de monoterpenos com atividade cardiovascular em bases de dados nacionais e internacionais.

\section{Metodologia}

Com o intuito de verificar o histórico de registros de patentes relacionadas à terpenos com atividade cardiovascular, efetuou-se a prospecção utilizando as seguintes bases de patentes depositadas: European Patent Office (Espacenet), World Intellectual Property Organization (WIPO), United States Patent and Trademark Office (USPTO) e Banco de Dados de Propriedade Industrial (INPI). A busca foi realizada utilizando-se as palavras-chave "terpeno e cardiovascular" e "terpene and cardiovascular" nos campos de pesquisa "título" e "resumo".

A pesquisa foi realizada durante o mês de julho de 2014, sendo analisados todos os pedidos de patentes existentes até o presente momento. Os documentos encontrados foram analisados sistematicamente, com o intuito de caracterizar o avanço tecnológico considerando o ano de depósito, classificação internacional de patentes (CIP), requerente da propriedade intelectual e país de depósito.

\section{Resultados e Discussão}

Com a realização da busca pelas bases de patentes citadas anteriormente, o maior número de patentes foi encontrado na base USPTO, de acordo com o observado na figura 1. 
Figura 1. Número de registro de patentes selecionadas nas bases de dados pesquisadas. WIPO (World Intellectual Property Organization), USPTO (United States Patent and Trademark Office), Espacenet (European Patent Office) e INPI (Banco de Dados de Propriedade Industrial).

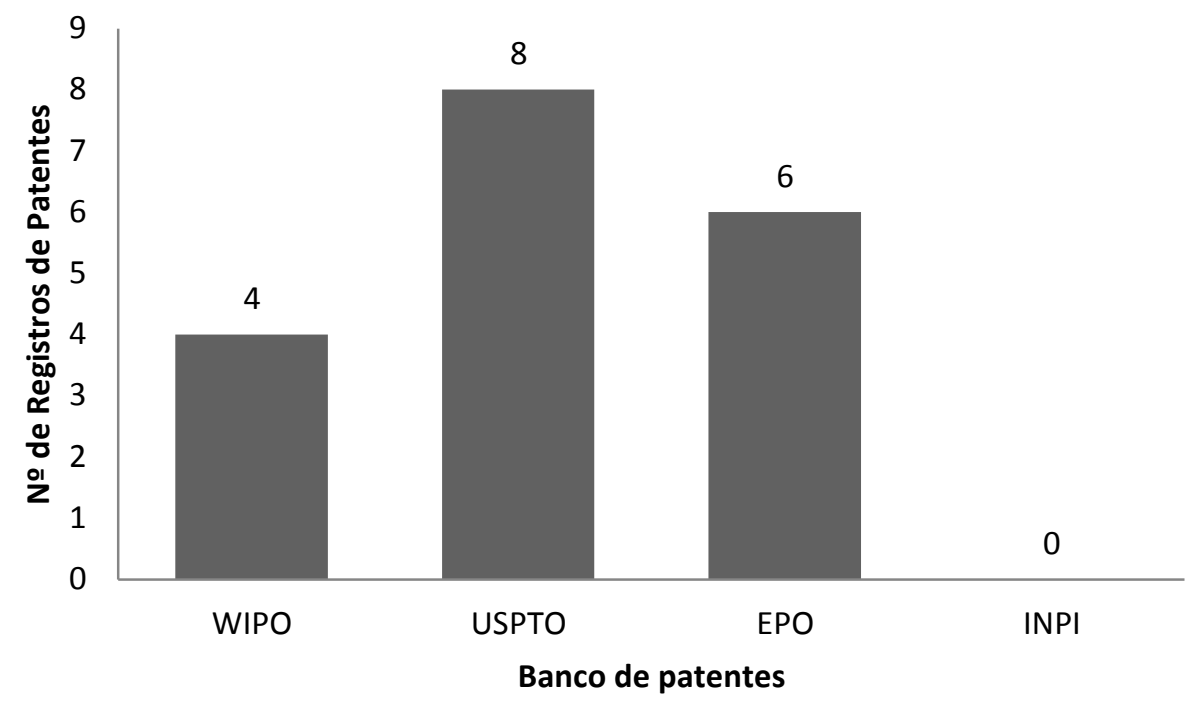

Com o intuito de avaliar a evolução temporal nos pedidos de patentes foi analisado o ano de depósito. Conforme observado na figura 2, é possível verificar que durante as décadas de 1980 e 1990 não ocorreram registros de patentes referente a atividade cardioprotetora. Porém em quatro anos ocorridos da década de 2010 observa-se a superação em relação aos registros de patentes ocorridos na década de 2000. Esse fato reflete a importância que tem sido atribuída à criação e proteção propriedade intelectual, bem como a interação entre instituições de ensino e pesquisa e empresas investidoras em insumos de inovação e tecnologia (TEIXEIRA et al., 2013, p. 123).

Figura 2.Número de patentes depositadas nos bancos de patentes de cada década dos últimos 44 anos.

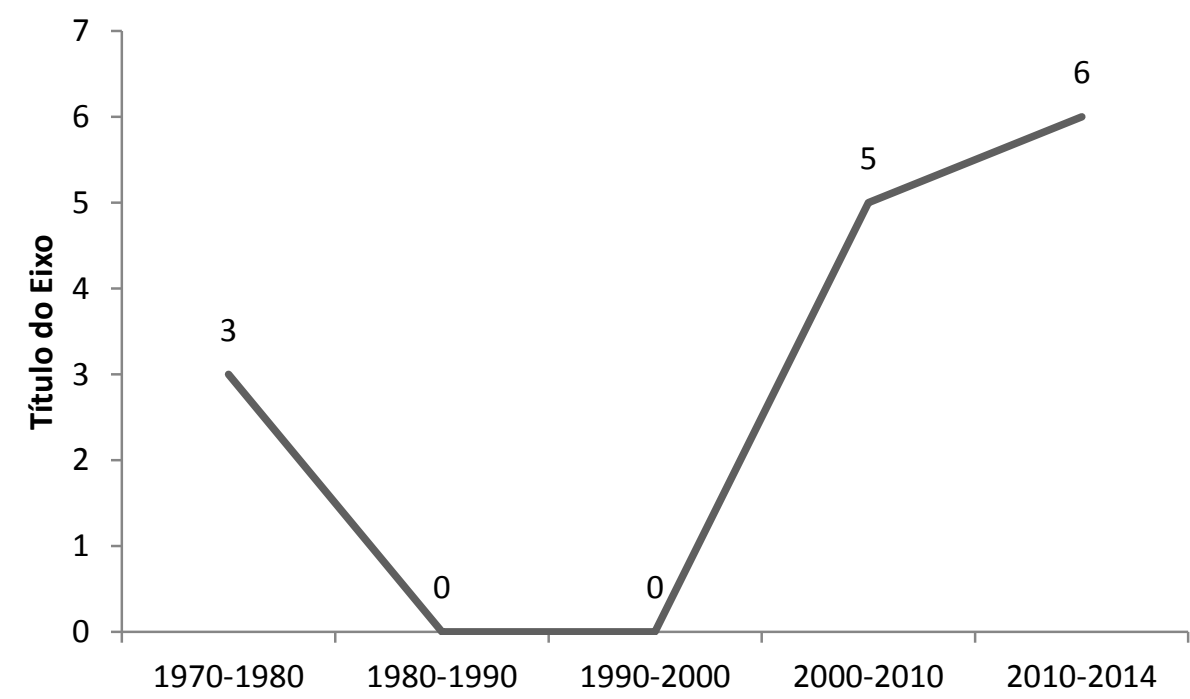

Para um melhor entendimento acerca da origem dos registros de patentes, a análise foi realizada com base em dados obtidos dos bancos de depósito citados anteriormente. Observando os dados obtidos, constata-se que Estados Unidos e China foram os maiores detentores de pedidos de 
patentes envolvendo terpenos com aplicação cardiovascular (figura 3). Resultado bastante semelhante também foi encontrado por Souza et al., (2014) onde os Estados Unidos também foi o país que mais pediu registro de terpenos com atividade biológica, enfatizando a sua liderança no setor de inovação e desenvolvimento de produtos farmacêuticos.

Figura 3. Depósitos de registros de patentes por países na base WIPO, EPO e USPTO. Sendo US (Estados Unidos). CN (China) e FR (França).

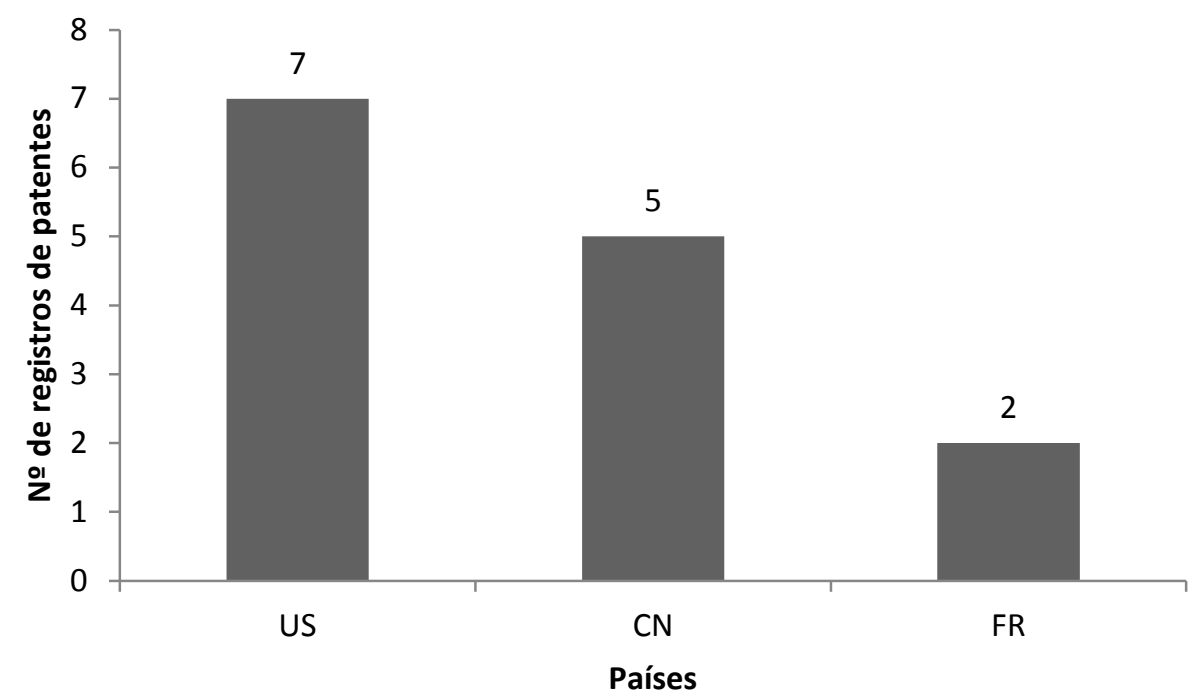

No que tange referência aos requerentes dos pedidos de patentes, as empresas são as que mais resguardam a propriedade intelectual, seguido de patentes de invenção de pessoas físicas e de instituições de ensino (Figura 4). De fato, existe fomentos à inovação tecnológica facilmente acessível nas empresas e no tema da propriedade intelectual voltados para a inovação (SILVA et al., 2013, p. 17).

Figura 4. Distribuição por requerente de origem dos depósitos de pedido de patentes encontradas nas bases. Sendo EMP ( Empresas), PF (Pessoa Física) e IE (Instituição de Ensino).

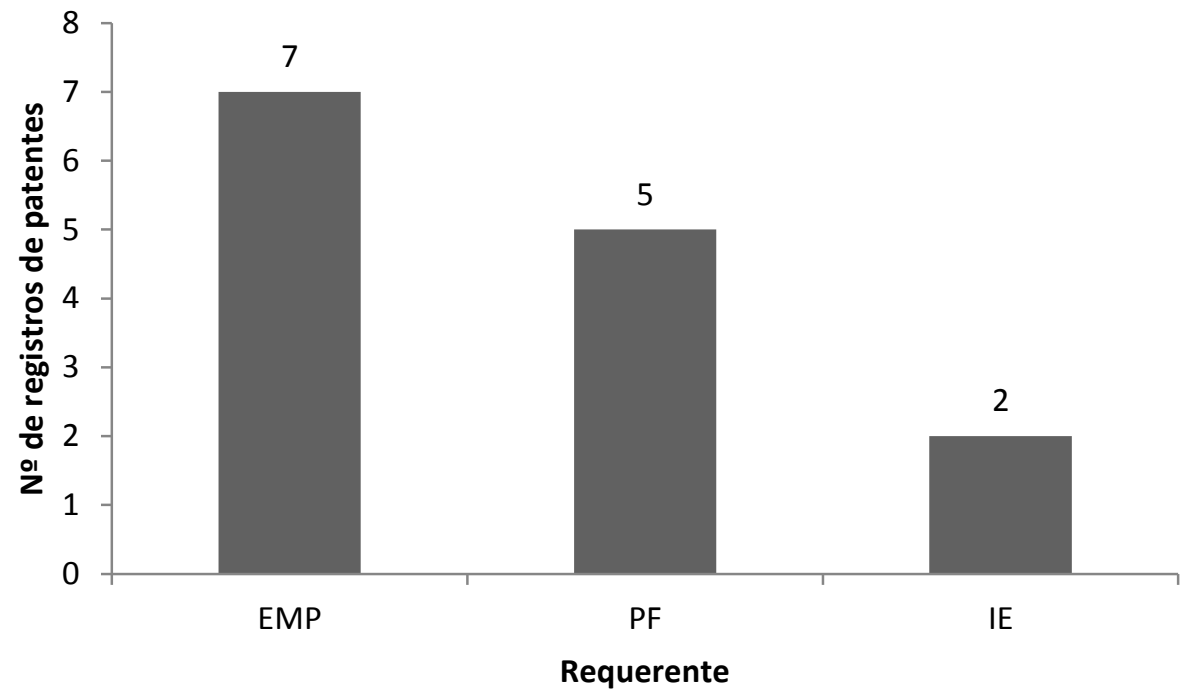


Quanto a avaliação de distribuição de patentes por Classificação Internacional de Patentes (CIP), muitos dos documentos encontrados estão classificados na subclasse A61K, que comporta aplicações para finalidades médicas, odontológicas e higiênicas (Figura 5). Visto que o interesse da pesquisa foi terpenos com aplicação cardiovascular, assim, as palavras chaves utilizadas auxiliam na busca direcionada as patentes para esta classificação.

Figura 5. Distribuição por código de classificação internacional (CIP) dos depósitos de pedidos de patentes encontradas nas bases. Sendo A61K = preparações para finalidades médicas, odontológicas ou higiênicas, C07= compostos químicos orgânicos.

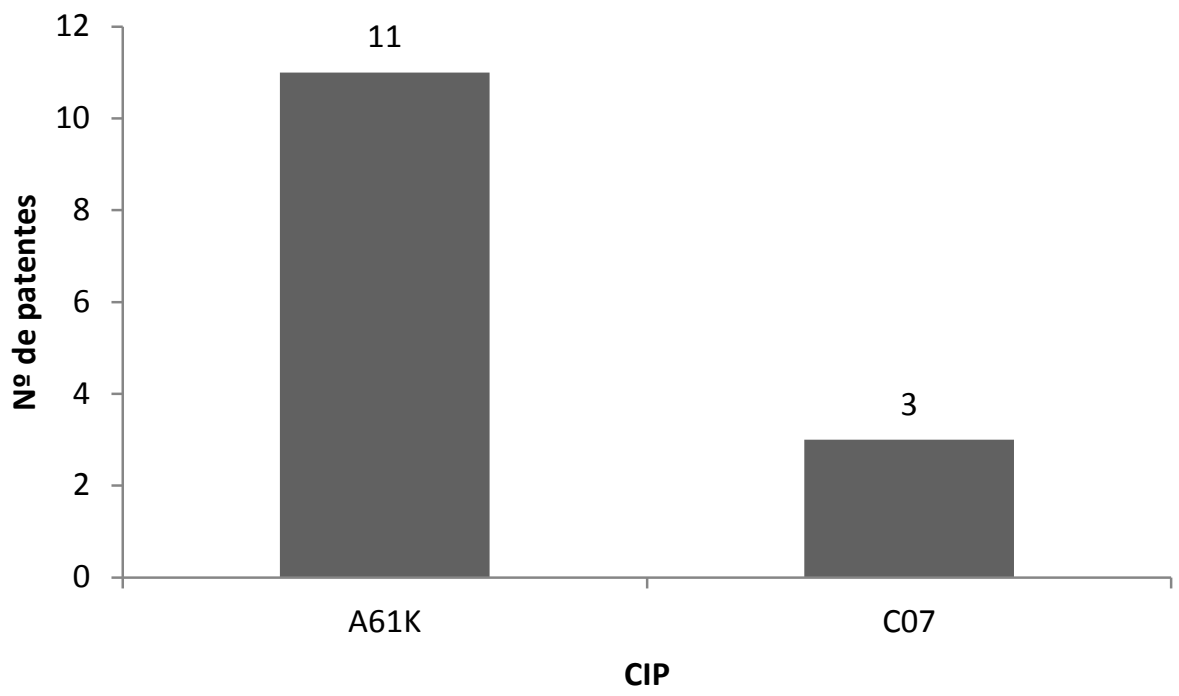

\section{Conclusão}

Com a análise nos bancos de patente consultados, foi possível observar que até o presente momento existem 14 registros de patentes que descrevem aplicações cardiovasculares de terpenos com perspectiva de desenvolvimento de produtos tecnológicos oriundos de novas alternativas terapêuticas para o controle de patologias cardiovasculares. Importante ressaltar que o Brasil não possui nenhum pedido de depósito referente a esse assunto sendo fatores prováveis à ausência de investimentos e fatores burocráticos envolvidos na proteção de propriedade intelectual.

\section{Referências}

AKINMOLADUN, A. C.; OLALEYE, M. T.; FAROMBI, E. O. Cardiotoxicity and Cardioprotective effects od African Medicianl Plants. Toxicologiacl survey of African medicinal plants, v.1, n.1 p.395-421, 2014.

ALMEIDA, R. A.; AGRA, M.F.; MAIOR, F. N. S.; SOUSA, D.P. Essential oils and their constituents: Anticonvulsivant activity: a review. Molecules, v. 16, p.2726-2742, 2011.

AYDIN, Y.; KUTLAY, O.; ARI, S.; DUMAN, S.; UZUNER, K.; AYDIN, S. Hypotensive effects of carvacrol on the blood pressure of normotensive rats. Planta Medical, v. 73, p.1365-1371, 2007. 
BASTOS, J. F.; MOREIRA, I. J.; RIBEIRO, T. P.; MEDEIROS, I. A.; ANTONIOLLI, A. R.; SOUSA, D. P.; SANTOS, M.R. Hypotensive and vasorelaxant effects of citronellol, a monoterpene alcohol, in rats. BasicalClinical Pharmacology and Toxicology, v. 106, p.331-337,2010.

CERQUEIRA, S. V. S.; GONDIN, A. N. S.; ROMAM-CAMPOS, D.; CRUZ, J. S.; PASSOS, A. G. S; LAUTONSANTOS, S.; LARA, A.; GUATIMOSIN, S.; CONDE-GARCIA, E.A.;OLIVEIRA, E.D.; VASCONCELOS, C. M. L. R-(+)-pulegoneimpairs $\mathrm{Ca}^{+}$homeostasis and causes negative inotropism in mammalianmyocardium. European Journal of Pharmacology, v. 672, p.135-142, 2011.

GIELEN, S.; LANDMESSER, U. The year in cardiology 2013: cardiovascular disease prevention. European heart journal, v.35, p. 307-312, 2014.

LOZANO, R., et al. Global and regional mortality from 235 causes of death for 20 age groups in 1990 and 2010: a sistematic analysis for the Global Burden of Disease Study 2010. Lancet, v. 380, n. 9859, p. 2095 - $2128,2012$.

MAGAlHÃES, P. J. C.; CRIDDLE, D. N.; TAVARES, R. A.; MELO, E. M.; MOTA, T. L.; LEAL-CARDOSO, J. H. Intestinal myorelaxant and antispasmodic effects of the essential oil of Croton nepetaefolius and its constituents cineole, methyl-eugenol and terpineol. Phytotherapy Research, v. 12, p. 172-177, 1998.

MATICH, A. J.; BUNN, B. J.; COMESKEY, D. J; HUNT, M. B.; ROWAN, D. D. Chirality and biosynthesis of lilac compounds in Actinidiaargutaflowers. Phytochemistry,v. 68, p.1746-1751, 2007.

PEIXOTO-NEVES, D.; SILVA-ALVES, K. S.; GOMES, M. D.; LIMA, F. C.; LAHLOU, S.; MAGALHÃES, P. J.; CECCATTO, V. M.; COELHO-DE-SOUZA, A. N.; LEAL-CARDOSO, J. H. Vasorelaxant effects of the monoterpenic phenol isomers, carvacrol and thymol, on rat isolated aorta. Fundamental Clinical Pharmacology, v. 24, p.341-350, 2010.

SANTOS, M. R. V.; MOREIRA, F. V.; FRAGA, B. P.; SOUSA, D. P.; BONJARDIN, L. R.; QUINTANS-JUNIOR, L. J. Cardiovascular effects of monoterpenes: a review. Brazilian Journal of Pharmacognozy, v. 21, n. 4, p.764-771, 2011.

SILVA, R.; MEDEIROS-FILHO, A. R.;PIRES, E. A.; SANTOS, A. B. Análise da evolução dos indicadores de produção científica e de produção tecnológica na Universidade Federal de Sergipe. RevistaGeintec, v. 3, n.5, p. 13-32, 2013.

SIQUEIRA, R.J.B.; MAGALHÃES, P.J.C.; LEAL-CARDOSO, J.H.; DUARTE, G.P.; LAHLOU, S. Cardiovascular effects of the essential oil of Croton zentnerileaves and its main constituents, anethole and estragole, in normotensive conscious rats. Life Sciences, v. 78, p. 2365-2372, 2006.

SOUZA, M. T. S.; GUIMARÃES, A. G.; ARAÚJO, A. A. S.; QUINTANS - JUNIOR, L. J. Monoterpenos com atividade anti-inflamatória: uma prospecção tecnológica. Revista Geintec, v. 4, n. 2, p. 867-875, 2014.

TEIXEIRA, R. C.; SOUZA, R. R. O uso das informações contidas em documentos de patentes nas práticas de inteligência competitiva: apresentação de um estudo de patentes da UFMG. Perspectivas em Ciência da Informação, v.18, n. 1, p. 106-125, 2013.

Recebido: 28/08/2014

Aprovado: 28/04/2015 